\title{
Analysis of infinite/finite unidirectional elastic phononic structures by BEM
}

\author{
H. F. Gao, T. Matsumoto, T. Takahashi \& H. Isakari \\ Nagoya University, Japan
}

\begin{abstract}
The transmission of elastic waves in infinite/finite unidirectional phononic crystals is investigated by using the boundary element method (BEM). For the infinite periodic structure, we use BEM to formulate a Bloch's eigenvalue problem which has a nonlinear property caused by the Hankel functions in the fundamental solution. This nonlinear eigenvalue problem is solved by employing a contour integral method and band gaps are found in the dispersion curves. For the finite structure, a certain number of layers for cells are given to connect the input and output domains. The numerical simulation shows that the finite structure also presents a frequency banded nature which coincides with the band gaps of the infinite structure.
\end{abstract}

Keywords: elastic wave, periodic structure, block SS method, boundary element method.

\section{Introduction}

The so-called phononic crystal is usually composed of materials with different properties in Young's modulus, density, Poisson's ratio, etc., and presents a frequency banded nature. This property makes the propagation of elastic waves in certain frequency range to be controlled by a certain collocation of the materials.

Many efforts have been made both on theoretical and experimental studies [1,2] for phononic crystals. In particular, for elastic wave propagation in an infinite 1D periodically layered media, the transfer matrix method is proposed to predict the dispersion curves across a specified frequency range $[3,4]$. The investigation of the existence of band gaps in quasi-one-dimensional phononic crystal plates with bounded unit cell is also reported [5]. 
Studies of the periodic structures of finite layers have also been made [6,7], in which the transfer matrix method is employed to predict the frequency-dependent transmission for a 1D finite periodic structure. Moreover, with the method of characteristics, propagation of waves in infinite and finite periodic structures have been investigated [7]. The latter study investigated the effects of the number of the layers for unit cells on the dynamics of a bounded structure [8,9]. A study of the finite elastic periodic materials and structures is presented with a perspective on both frequency and temporal domains in [10], in which the correlation between the dynamic response of the respective finite and infinite systems is discussed.

In this paper, we use the BEM to study the transmission of elastic waves in a finite structure having periodic part that can be considered as a phononic crystal plate. When using BEM, only the boundary is discretized into line meshes in the 2D problem. We investigate the dispersion relation of the infinite structures by giving the periodic boundary condition to a unit cell. The nonlinear Bloch eigenvalue problem resulting from the use of BEM is solved by using the block Sakurai-Suigura (SS) method [11]. In this work, the longitudinal and transverse waves are considered, and a traction free boundary condition is given on the surfaces of the phononic crystal plates. Numerical simulation demonstrates that the reduction of the transmission shows a good agreement with the band gaps obtained by the block SS method.

\section{Formulations}

\subsection{The boundary element method for 2D elastodynamic problem}

The governing equation for a harmonic vibration of structure of homogeneous and isotropic material, represented by displacements, is

$$
\left(C_{1}^{2}-C_{2}^{2}\right) u_{j, j k}(x, t)+C_{2}^{2} u_{k, j j}(x, t)=\ddot{u}_{k}(x, t),
$$

where $C_{1}$ and $C_{2}$ are the wave speeds of longitudinal and transverse waves, respectively, written as,

$$
\begin{aligned}
& C_{1}=\sqrt{(\lambda+2 \mu) / \rho}=\sqrt{E(1-\nu) /[\rho(1+\nu)(1-2 \nu)]}, \\
& C_{2}=\sqrt{\mu / \rho}=\sqrt{E /[2 \rho(1+\nu)]},
\end{aligned}
$$

where $\rho$ is the density of the medium, $\lambda$ and $\mu$ are Lamé's constants, $E$ is Young's modulus, and $\nu$ is Poisson's ratio.

For harmonic vibrations, the displacement can be written in a time-independent form:

$$
u_{i}(x, t)=U_{i}(x, \omega) e^{\mathrm{i} \omega t},
$$

where i denotes the imaginary unit, $\omega=2 \pi f$ is the circular frequency, and $f$ is the frequency. 
Substituting Eq. (4) into Eq. (1), we obtain the time-harmonic form of the governing equation, as follows:

$$
\left(C_{1}^{2}-C_{2}^{2}\right) U_{j, j k}(x, \omega)+C_{2}^{2} U_{k, j j}(x, \omega)+\omega^{2} U_{k}(x, \omega)=0 .
$$

The boundary integral equation corresponding to the above boundary value problem is obtained as

$$
\begin{gathered}
c_{k l}(y) U_{k}(y, \omega)+\int_{\Gamma} t_{k l}^{*}(x, y, \omega) U_{k}(x, \omega) \mathrm{d} \Gamma(x) \\
-\int_{\Gamma} u_{k l}^{*}(x, y, \omega) T_{k}(x, \omega) \mathrm{d} \Gamma(x)=0,
\end{gathered}
$$

where $c_{k l}$ depends on the geometry of the boundary on which $y$ is located, the kernels $u_{i j}^{*}(x, y)$ and $t_{i j}^{*}(x, y)$ are known as the displacement and traction fundamental solutions which are given for the two-dimensional case [12] as

$$
\begin{gathered}
u_{i j}^{*}(x, y)=\frac{1}{\alpha \pi \rho C_{2}^{2}}\left[\psi \delta_{i j}-\chi r_{, i} r_{, j}\right] \\
t_{i j}^{*}(x, y)=\frac{1}{\alpha \pi}\left[\left(\frac{d \psi}{d r}-\frac{1}{r} \chi\right)\left(\delta_{i, j} \frac{\partial r}{\partial n}+r_{, j} n_{i}\right)-\frac{2}{r} \chi\left(n_{j} r_{, j}\right.\right. \\
\left.\left.-2 r_{, i} r_{, j} \frac{\partial r}{\partial n}\right)-2 \frac{d \chi}{d r} r_{, i} r_{, j} \frac{\partial r}{\partial n}+\left(\frac{C_{1}^{2}}{C_{2}^{2}}-2\right)\left(\frac{\psi}{d r}-\frac{d \chi}{d r}-\frac{\alpha}{2 r} \chi\right) r_{, i} n_{j}\right],
\end{gathered}
$$

where

$$
\begin{aligned}
& \psi=K_{0}\left(\frac{s r}{C_{2}}\right)+\frac{C_{2}}{s r}\left[K_{1}\left(\frac{s r}{C_{2}}\right)-\frac{C_{2}}{C_{1}} K_{1}\left(\frac{s r}{C_{1}}\right)\right], \\
& \chi=K_{2}\left(\frac{s r}{C_{2}}\right)-\frac{C_{2}^{2}}{C_{1}^{2}} K_{2}\left(\frac{s r}{C_{1}}\right),
\end{aligned}
$$

and $s=\mathrm{i} \omega, \alpha=2$ for the $2 \mathrm{D}$ case, and $K_{0}, K_{1}$, and $K_{2}$ are the modified Bessel functions of order 0,1 , and 2 , respectively.

Discretizing Eq. (6) with $N$ constant boundary elements, we obtain a linear equation as follows:

$$
\begin{gathered}
c_{k l}(y) U_{k}^{i}(y, \omega)+\sum_{j=1}^{N}\left(\int_{\Gamma_{j}} t_{k l}^{*}(x, y, \omega) \mathrm{d} \Gamma(x)\right) U_{k}^{j}(x, \omega) \\
-\sum_{j=1}^{N}\left(\int_{\Gamma_{j}} u_{k l}^{*}(x, y, \omega) \mathrm{d} \Gamma(x)\right) T_{k}^{j}(x, \omega)=0,
\end{gathered}
$$

where $c_{k l}=(1 / 2) \delta_{k l}$ when the boundary on which $y$ lies is smooth, and $U_{k}^{j}(y, \omega)$ and $T_{k}^{j}(x, \omega)$ denote the $x_{k}$ component of the displacement and traction on the element $S_{j}$. 
Let $i$ vary from 1 to $N$, then we obtain a $2 N$ system of linear algebraic equations for $\mathbf{U}$ and $\mathbf{T}$ as

$$
\mathbf{B U}=\mathbf{G T}
$$

where $\mathbf{B}$ and $\mathbf{G}$ are $2 N \times 2 N$ matrices, respectively, and $\mathbf{U}, \mathbf{T} \in \mathbb{C}^{2 N}$.

\subsection{The block SS method for the Bloch eigenvalue problem for infinite phononic crystals}

For infinite periodic structures, by applying the periodic boundary condition and homogeneous boundary condition to a unit cell, we have a nonlinear eigenvalue problem, as follows:

$$
\mathbf{F}(\omega, k) \mathbf{X}=\mathbf{0} .
$$

For phononic crystals in uni-directional periodicity, the wave-number vector becomes a scalar $k$ and is set to vary from 0 to $\pi / d$, and the dispersion curves can be obtained by solving eigenvalues for $\omega$ determined by Eq. (13).

To solve this highly nonlinear eigenvalue problem, we use the block SS method and convert it to a generalized eigenvalue problem. The block SS method provides an eigenvalue problem of a linear matrix pencil of Hankel matrices:

$$
\mathbf{H}_{K \hat{l}}^{<}-\omega \mathbf{H}_{K \hat{l}}=\mathbf{0},
$$

where $\mathbf{H}_{K \hat{l}}^{<}$and $\mathbf{H}_{K \hat{l}}$ are Hankel matrices defined as follows:

$$
\begin{array}{r}
\mathbf{H}_{K \hat{l}}=\left(\begin{array}{cccc}
\mathbf{M}_{0} & \mathbf{M}_{1} & \cdots & \mathbf{M}_{K-1} \\
\mathbf{M}_{1} & \mathbf{M}_{2} & \cdots & \mathbf{M}_{K} \\
\vdots & \vdots & \ddots & \vdots \\
\mathbf{M}_{K-1} & \mathbf{M}_{K} & \cdots & \mathbf{M}_{2 K-2}
\end{array}\right), \\
\mathbf{H}_{K \hat{l}}^{<}=\left(\begin{array}{cccc}
\mathbf{M}_{1} & \mathbf{M}_{2} & \cdots & \mathbf{M}_{K} \\
\mathbf{M}_{2} & \mathbf{M}_{3} & \cdots & \mathbf{M}_{K+1} \\
\vdots & \vdots & \ddots & \vdots \\
\mathbf{M}_{K} & \mathbf{M}_{K+1} & \cdots & \mathbf{M}_{2 K-1}
\end{array}\right),
\end{array}
$$

where $\mathbf{M}_{m}$ is the moment matrix defined with a contour integral along a closed Jordan curve $S$ in the complex plane of $\omega$ :

$$
\mathbf{M}_{m}=\frac{1}{2 \pi \mathrm{i}} \oint_{S} \mathbf{U}^{H} \mathbf{F}^{-1}(z) \mathbf{V} z^{m} \mathrm{~d} z
$$

where $(\cdot)^{H}$ denotes the conjugate transpose, $\mathbf{V}$ is a $2 N \times \hat{l}$ matrix formed by column vectors $\mathbf{v}_{1}, \mathbf{v}_{2}, \ldots, \mathbf{v}_{\hat{l}} \in \mathbb{C}^{2 N}$, and for simplicity, $\mathbf{U}=\mathbf{V}$.

Using Eq. (14), we can extract the eigenvalues located within $S$. 


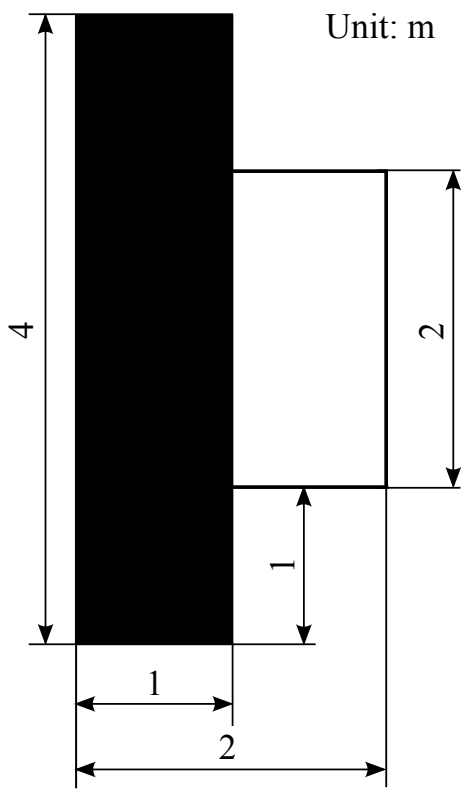

Figure 1: The unit cell of the periodic structure. The stiff/dense material and the compliant/light material phases are shown in black and white, respectively.

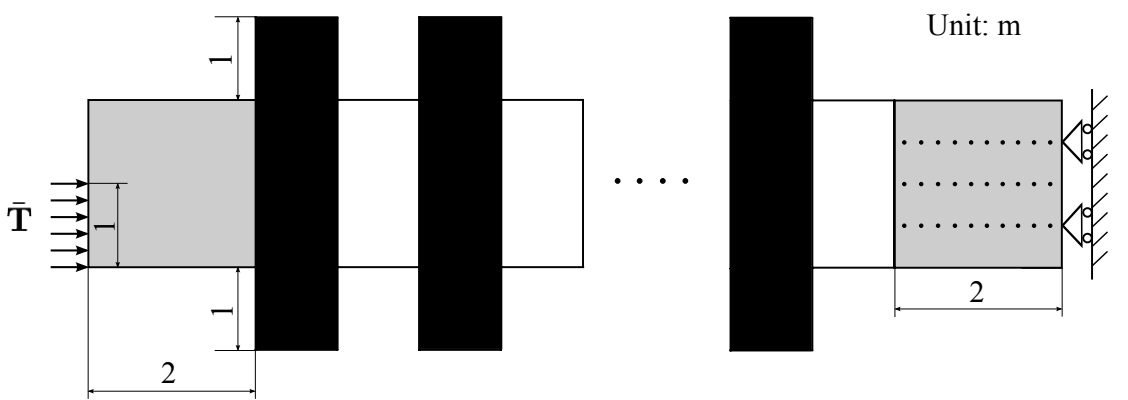

Figure 2: Two square domains are connected with the periodic cells of $N_{L}$ layers. The input and output domains are the left and right square domains shaded in gray, respectively.

\section{Numerical simulation}

A unit cell composed of alternating layers consisting of two different materials is shown in Fig. 1, where the region shown in black corresponds to a stiff and dense material (denoted by subscript ' 2 ') of density $\rho_{2}=1.6 \times 10^{4}\left[\mathrm{~kg} / \mathrm{m}^{3}\right]$, Young's modulus $E_{2}=1.248 \times 10^{8}[\mathrm{~Pa}]$, and Poisson's ratio $\nu_{2}=0.34$, while the region 


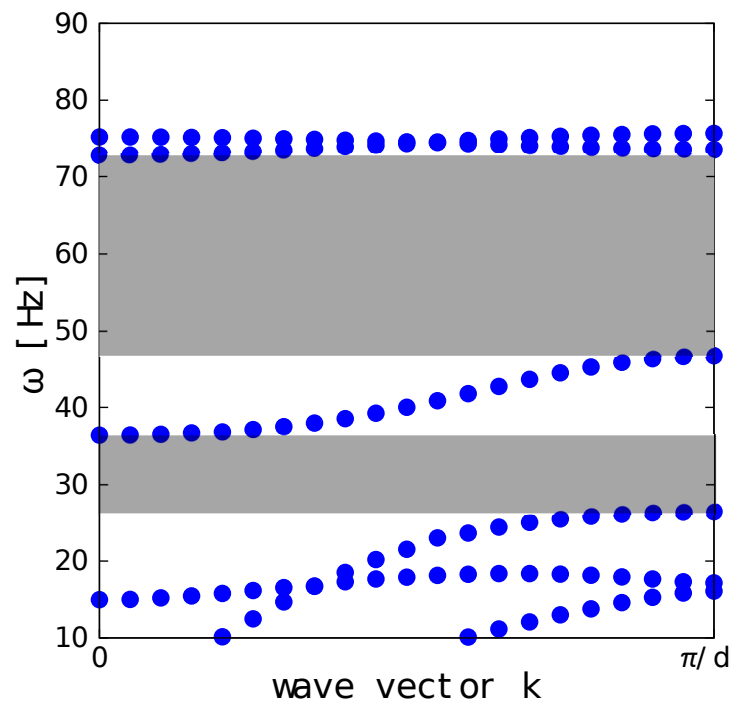

Figure 3: The band structure for the infinite structure. Shaded ranges denote the concerned band gaps.

in white corresponds to a compliant and light material (denoted by subscript ' 1 ') of density $\rho_{1}=2.0 \times 10^{3}\left[\mathrm{~kg} / \mathrm{m}^{3}\right]$, Young's modulus $E_{1}=7.8 \times 10^{6}[\mathrm{~Pa}]$, and Poisson's ratio $\nu_{1}=0.34$.

The finite structure is considered as comprising of the input domain, output domain, and $N_{L}$ layers of cells, as depicted in Fig. 2, where an excitation $\overline{\mathbf{T}}=\{1.0,0.0\}[\mathrm{N} / \mathrm{m}]$ is given on the left square region in gray, which is considered as the input domain. Roller support boundary condition is assumed on the right-end boundary of the right-side square region shown in gray color. This square region is considered as the output domain. The traction free boundary condition is given on the rest of the boundaries. The material properties of those of the input and output domains are assumed to be as those of material 2. In the output domain, we consider 30 observation points at which the displacements are calculated. We define a cartesian coordinate system whose origin is placed at the left bottom corner of the output square domain. Then, the coordinates of the observation points are $(0.1,1),(0.3,1), \ldots,(1.9,1)$ from point 1 to $10,(0.1,0.5),(0.3,0.5), \ldots,(1.9,0.5)$ from point 11 to 20 , $(0.1,1.5),(0.3,1.5), \ldots,(1.9,1.5)$ from point 21 to 30 .

Firstly, let us see the band structure shown by the dispersion curves in Fig. 3, where the circular frequency range is chosen as $10 \sim 90[\mathrm{~Hz}]$. It should be noted that the band structure is determined by an infinite system with the unit cell shown in Fig. 1. Here, we consider only the two band gaps represented by the shaded ranges. 


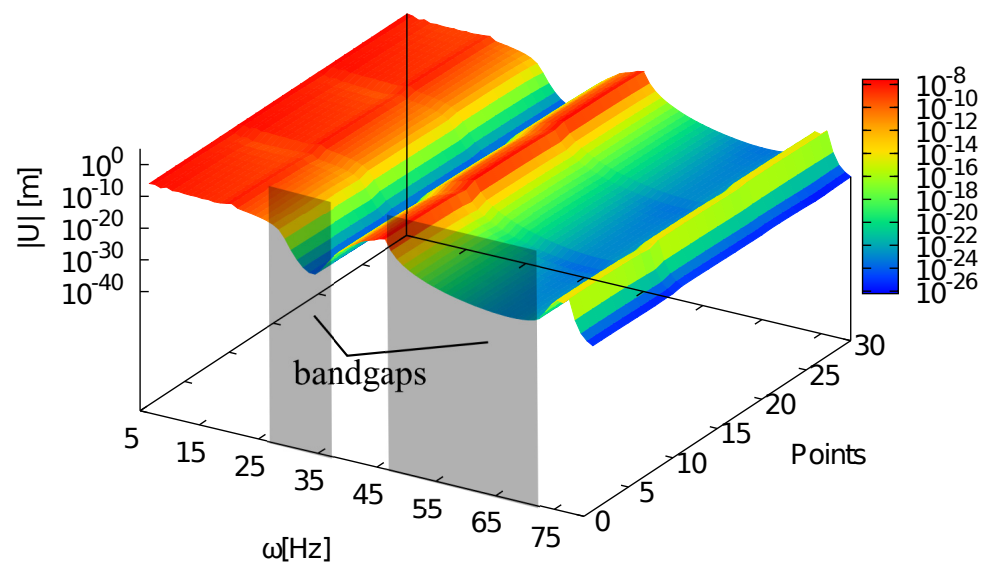

(a)

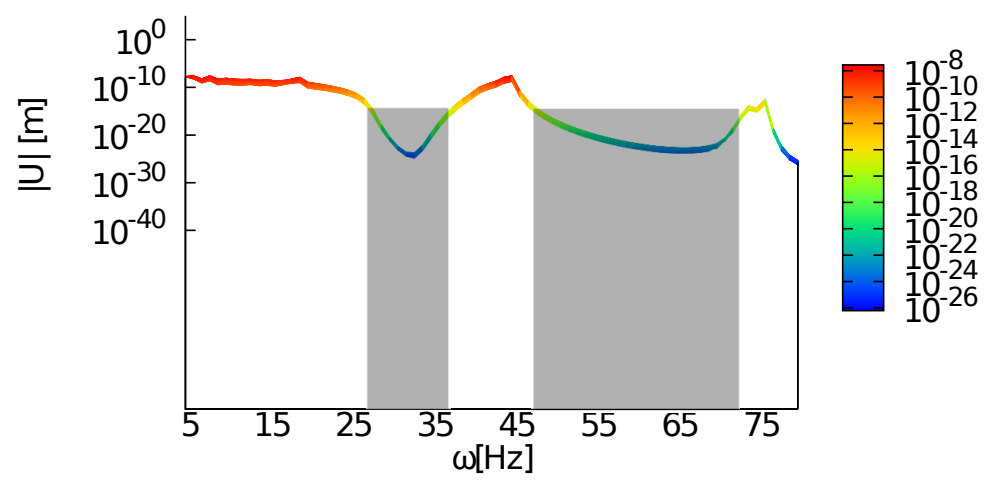

(b)

Figure 4: (a) The transmission of $|\mathbf{U}|$ of the structure with 20 periodic layers and (b) the projection to the $\omega-|\mathbf{U}|$ plane.

For the finite structure, we show in Figs. 4 and 5 the displacement responses $|\mathbf{U}|=\sqrt{U_{x}^{2}+U_{y}^{2}}$ of the observation points in the output domain with $N_{L}=20$ and $N_{L}=40$ layers of cells, and its projection to the $\omega$ - $|\mathbf{U}|$ plane, respectively. It can be seen that the displacement transmitting to the output domain becomes smaller with the increase in the number of layers.

\section{Concluding remarks}

In this work, the band structure of a unidirectional infinite phononic structure and the displacement transmission in a finite structure are investigated by using the BEM and the block SS method. The eigenvalues of the Bloch eigenvalue problem of the infinite structure are extracted by using the block SS method. The band gaps 


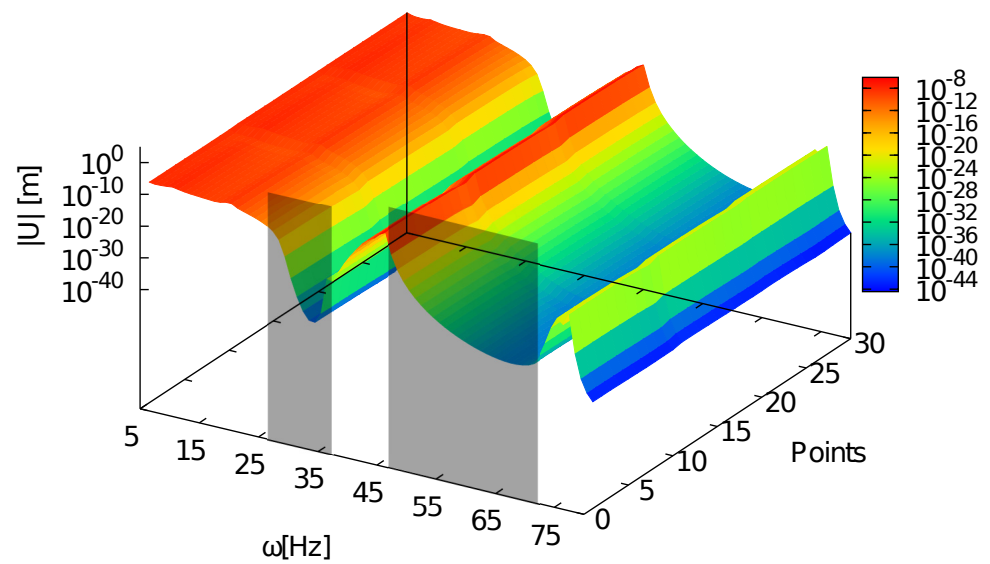

(a)

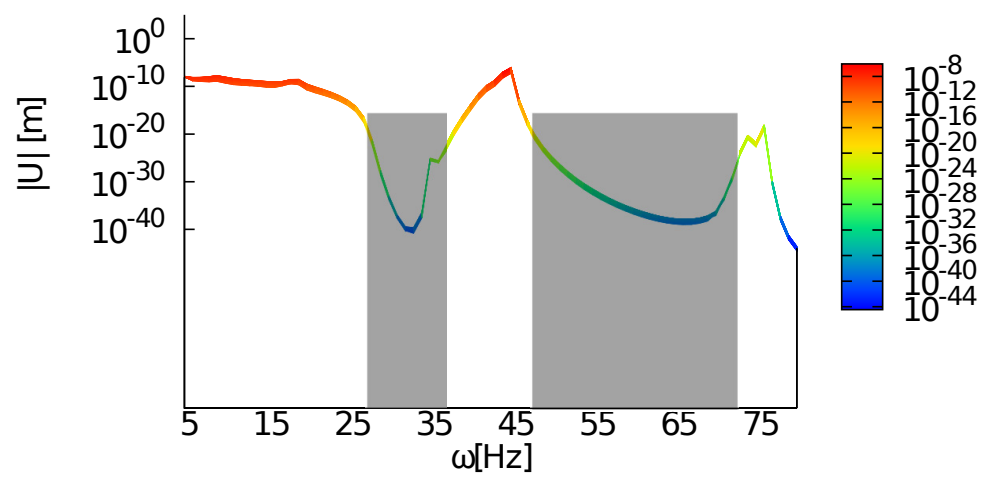

(b)

Figure 5: (a) The transmission of $|\mathbf{U}|$ of the structure with 40 periodic layers and (b) the projection to the $\omega$-|U $\mathbf{U} \mid$ plane.

of the infinite periodic structure are found by plotting the dispersion curves. The transmission of the elastic waves for the finite periodic structure with a certain number of layers of periodic cells is computed, and it is found that the reduction of the transmission occurs at the band gaps of the infinite structure and can be enhanced by increasing the number of layers for cells.

\section{Acknowledgements}

The authors are grateful for financial support from JSPS KAKENHI Grant Number 25289022, the China Scholarship Council (CSC) file No. 2009612004, and Nagoya University during the preparation of this work. 


\section{References}

[1] Kushwaha MS, Halevi P, Dobrzynski L, Djafari-Rouhani B. Acoustic bandstructure of periodic elastic composites. Phys Rev B 1993; 71: 2022-2025.

[2] Sigalas M, Economou EN. Band-structure of elastic-waves in 2-dimensional systems. Solid State Commun 1993; 86: 141-143.

[3] Esquivel-Sirvent R, Cocoletzi GH. Band-structure for the propagation of elastic-waves in superlattices. J Acoust Soc Am 1994; 95: 86-90.

[4] Shen MR, Cao WW. Acoustic bandgap formation in a periodic structure with multilayer unit cells. J Phys D Appl Phys 2000; 33: 1150-1154.

[5] Wang XF, Kushwaha MS, Vasilopoulos P. Tunability of acoustic spectral gaps and transmission in periodically stubbed waveguides. Phys Rev B 2001; 65: 035107.

[6] Cao WW, Qi WK. Plane-wave propagation in finite 2-2-composites. J Appl Phys 1995; 78: 4627-4632.

[7] Bedford A. Drumheller DS. Introduction to Elastic Wave Propagation. Wiley, Chichester, 1994.

[8] Jensen JS. Phononic band gaps and vibrations in one- and two-dimensional mass-spring structures. J Sound Vib 20003; 266: 1053-1078.

[9] Hussein MI, Hulbert GM, Scott RA. Band-gap engineering of elastic wave guides using periodic materials, Proc of the 2003 ASME International Mechanical Engineering Congress and $R \& D$ Expo, Washington, DC. ASME Publications, New York, 2003: 799-807.

[10] Hussein MI, Hulbert GM, Scott RA. Dispersive elastodynamics of 1D banded materials and structures: analysis. J Sound Vib 2006; 289: 779-806.

[11] Asakura J, Sakurai T, Tadano H, Ikegami T, Kimura K. A numerical method for nonlinear eigenvalue problems using contour integrals. JSIAM Lett 2009; 1: 52-55.

[12] Cruse TA. A direct formulation and numerical solution of the general transient elastodynamic problem. J Math Anal Appl 1968; 22: 244-259. 